\title{
A molecular portrait of gastrointestinal stromal tumors: an integrative analysis of gene expression profiling and high-resolution genomic copy number
}

\author{
Annalisa Astolfi ${ }^{1}$, Margherita Nannini ${ }^{2}$, Maria Abbondanza Pantaleo ${ }^{1,2}$, Monica Di Battista ${ }^{2}$, Michael C Heinrich ${ }^{3}$, \\ Donatella Santini ${ }^{4}$, Fausto Catena ${ }^{5}$, Christopher L Corless ${ }^{6}$, Alessandra Maleddu², Maristella Saponara ${ }^{2}$, \\ Cristian Lolli ${ }^{2}$, Valerio Di Scioscio ${ }^{7}$, Serena Formica ${ }^{1}$ and Guido Biasco ${ }^{1,2}$
}

In addition to KIT and PDGFRA mutations, sequential accumulation of other genetic events is involved in the development and progression of gastrointestinal stromal tumors (GISTs). Until recently, the significance of these other alterations has not been thoroughly investigated. We report the first study that integrates gene expression profiling and high-resolution genomic copy number analyses in GIST. Fresh tissue specimens from 25 patients with GIST were collected, and gene expression profiling and high-resolution genomic copy number analyses were performed, using Affymetrix U133Plus and SNP array 6.0. We found that all 21 mutant GIST patients showed both macroscopic cytogenetic alterations and cryptic microdeletions or amplifications, whereas $75 \%$ (three of four) of wild-type patients with GIST did not show genomic imbalances. The most frequently observed chromosomal alterations in patients with mutant GIST included $14 q$ complete or partial deletion (17 of 25), $1 p$ deletion (14 of 25) and 22q deletion (10 of 25). Genetic targets of the chromosomal aberrations were selected by integrated analysis of copy number and gene expression data. We detected the involvement of known oncogenes and tumor suppressors including KRAS in chr $12 \mathrm{p}$ amplification and KIF1B, PPM1A, NF2 in chr 1p, 14q and 22p deletions, respectively. The genomic segment most frequently altered in mutated samples was the $14 q 23.1$ region, which contains potentially novel tumor suppressors, including DAAM1, RTN1 and DACT1. siRNA-mediated RTN1 downregulation showed evidence for the potential role in GIST pathogenesis. The combination of gene expression profiling and high-resolution genomic copy number analysis offers a detailed molecular portrait of GISTs, providing an essential comprehensive knowledge necessary to guide the discovery of novel target genes involved in tumor development and progression.

Laboratory Investigation (2010) 90, 1285-1294; doi:10.1038/labinvest.2010.110; published online 14 June 2010

KEYWORDS: gastrointestinal stromal tumors; gene expression profiling; SNPs array

Gastrointestinal stromal tumors (GISTs) are the most common mesenchymal tumors of the gastrointestinal tract. These are characterized by mutually exclusive KIT or PDGFR- $\alpha$ (PDGFRA) gain-of-function mutations, leading to constitutive ligand-independent activation of each receptor's signaling pathways. $^{1,2}$ The most frequently identified mutations include KIT exon 11 point mutations (65-70\%), KIT exon 9 point mutations (10-20\%), KIT exon 13 point mutations (1-2\%), KIT exon 17 point mutations $(<1 \%)$, PDGFRA exon 18 point mutations (6-7\%), PDGFRA exon 12 point mutations $(<1 \%)$ and PDGFRA exon 14 point mutations $(0.5 \%)$. However, approximately $10-15 \%$ of GISTs are defined as wild type (WT) as they lack both KIT and PDGFRA mutations. ${ }^{3}$ The primary mutational status of KIT and PDGFRA in a patient has a predictive value of responsiveness to tyrosine kinase inhibitors (TKIs). Furthermore,

\footnotetext{
'Interdepartmental Centre for Cancer Research 'G. Prodi', University of Bologna, Bologna, Italy; ${ }^{2}$ Department of Hematology and Oncological Sciences 'L. A. Seràgnoli', S. Orsola-Malpighi Hospital, University of Bologna, Bologna, Italy; ${ }^{3}$ Department of Medicine, Portland VA Medical Center and Oregon Health and Science University Knight Cancer Institute, Oregon Health and Science University, Portland, OR, USA; ${ }^{4}$ Pathology Unit, S. Orsola-Malpighi Hospital, University of Bologna, Bologna, Italy; ${ }^{5}$ Emergency Surgery and Transplant Department, S. Orsola-Malpighi Hospital, University of Bologna, Bologna, Italy; ${ }^{6}$ Department of Pathology, Portland VA Medical Center and Oregon Health and Science University Knight Cancer Institute, Oregon Health and Science University, Portland, OR, USA and ${ }^{7}$ Division of Pneumo-Nefro, Department of Radiology, University Hospital S. Orsola-Malpighi, University of Bologna, Bologna, Italy

Correspondence: Dr MA Pantaleo, MD, PhD, Department of Hematology and Oncological Sciences, 'L. A. Seràgnoli', S. Orsola-Malpighi Hospital, University of Bologna, Via Massarenti 9, 40138 Bologna, Italy.
}

E-mail: maria.pantaleo@unibo.it

Received 20 January 2010; revised 10 March 2010; accepted 29 March 2010 
acquisition of new point mutations during disease progression represents the most common mechanism of resistance to TKIs. ${ }^{4-6}$

In addition to KIT and PDGFRA mutations, sequential accumulation of other genetic events may be involved in the development and progression of GIST. ${ }^{7}$ Loss of chromosomes 14 and 22 is the most frequently described genetic aberration in GISTs, regardless of tumor genotype. These chromosomal losses may represent an underlying pathogenetic event resulting in the inactivation or haploinsufficiency of tumor suppressor genes. ${ }^{8-20}$ However, the biologic significance of these genetic alterations, as well as their clinical implications, remains unknown. To address this issue, we performed an integrative analysis of gene expression profiling and high-resolution genomic copy number in 25 GIST samples to investigate the relationship between karyotype and gene expression profile, and to identify new haploinsufficient tumor suppressor genes involved in GIST pathogenesis and tumor progression.

\section{MATERIALS AND METHODS}

Fresh tissue specimens of GISTs from 25 adult patients were collected, snap-frozen in liquid nitrogen and stored at $-80^{\circ} \mathrm{C}$. Patient characteristics are listed in Table 1.

Mutational analysis of KIT (exons 9, 11, 13 and 17) and PDGFRA (exons 12, 14 and 18) revealed the following: 14 patients had KIT gain-of-function mutations (13 in exon 11 and 1 in exon 9), 7 patients had PDGFRA activating mutation ( 3 in exon 12, 1 in exon 14 and 4 in exon 18) and 4 patients had WT disease.

\section{SNP Array}

Genomic DNA was extracted using the QIAamp DNA kit (Qiagen), labeled and hybridized to an oligonucleotide SNP array (Genome Wide SNP 6.0; Affymetrix), which investigates 1.8 million markers (SNP and CNV probes) on all chromosomes. Quality control was performed by Contrast QC and MAPD calculation. Copy number analysis was performed by Partek Genomics Suite, which compares Signal $\log _{2}$ ratios to a reference of 270 normal Hapmap samples. Genomic segmentation was used to detect amplified and deleted segments with stringent parameters $(P<0.0001,>20$ markers, signal $/$ noise $\geqslant 0.6$, minimal region size $=100 \mathrm{mar}-$ kers) To control for hyperfragmentation adjacent segments separated by $<50$ probes were combined into one single segment, and only segments $>100$ probes were considered. Multiple hypothesis correction by Benjamini and Hochberg and by cghFLasso algorithm ${ }^{21}$ was applied and FDR threshold was set at 0.05 . Correspondence with gene expression was calculated by Spearman's (rank) correlation coefficient. SNP, gene and cytogenetic band locations are based on the hg18 Genome build. $\chi^{2}$-Statistics was used to determine the correlation between copy number alterations (CNAs) and mutated phenotype.

\section{Gene Expression}

Cellular RNA was extracted using RNeasy Spin columns (Qiagen), quality-controlled and labeled as directed by the Affymetrix expression technical manual before hybridization to U133Plus 2.0 arrays. Gene expression data were quantified by the RMA algorithm, filtered and analyzed with supervised techniques by Limma modified $t$-test ${ }^{22}$ and corrected for multiple testing by Benjamini and Hochberg method for the detection of differentially expressed genes. Gene expression and differential $P$-value on chromosome 14 were plotted against chromosomal position. Genes mapping in minimal overlapping regions in more than four samples were crossreferenced on the CancerGene resource (http://cbio.mskcc. org/CancerGenes). Oncogenes and tumor suppressor genes were selected only if showing a high correlation between copy number state and gene expression $(P$-value of correlation $<0.05)$.

Gene expression and SNP array raw data were submitted to the GEO database with the identifier GSE20710.

\section{Quantitative PCR (qPCR)}

Total RNA was reverse transcribed using Superscript II (Invitrogen Life Technologies) with oligo-dT primers, according to the manufacturer's guidelines. Gene-specific primers were designed with Primer Express 3.0 Software (Applied Biosystems) and qPCR was performed using FastStart Sybr Green (Roche) on the LightCycler 480 apparatus (Roche). DDCt method was used to quantify gene product levels relative to the GAPDH housekeeping gene. Significance was estimated by the Mann-Whitney $U$-test.

\section{Cell Lines and siRNA Gene Knockdown}

GIST882 cells (kind gift from Professor Jonathan A Fletcher, Harvard Medical School, Boston, MA, USA) were cultured in RPMI $+15 \%$ FBS. This cell line harbors an exon 13 missense mutation, resulting in a single amino-acid substitution, K642E. Cells $\left(2 \times 10^{6}\right)$ were transfected with $100 \mathrm{pmol}$ Hs_RTN1_5 and Hs_RTN1_6 HP siRNA (Qiagen) or nontargeting pooled siRNA (Dharmacon, Thermo Scientific) by Amaxa Electroporator Nucleofector (Lonza, Basel, Switzerland) using the Mirus Ingenio Electroporation solution (Mirus Bio LLC, Madison, WI, USA). After $4 \mathrm{~h}$ of incubation cells were seeded in 96-well plates in triplicate. Cell growth was measured after $72 \mathrm{~h}$ of incubation with WST1 cell proliferation reagent assay (Roche).

\section{RESULTS}

\section{Recurrent Genomic Copy Number Alterations in GIST}

We analyzed genomic CNAs in 25 GIST samples by highresolution SNP 6.0 mapping arrays. A genome-wide view of the frequency of copy number changes is shown in Figure 1a. The most relevant alterations are reported in Supplementary Table S1. GIST samples are very heterogeneous about the number and size of karyotypic alterations. The majority of patients showed few genomic imbalances, mainly as whole 
Table 1 Patients' characteristics

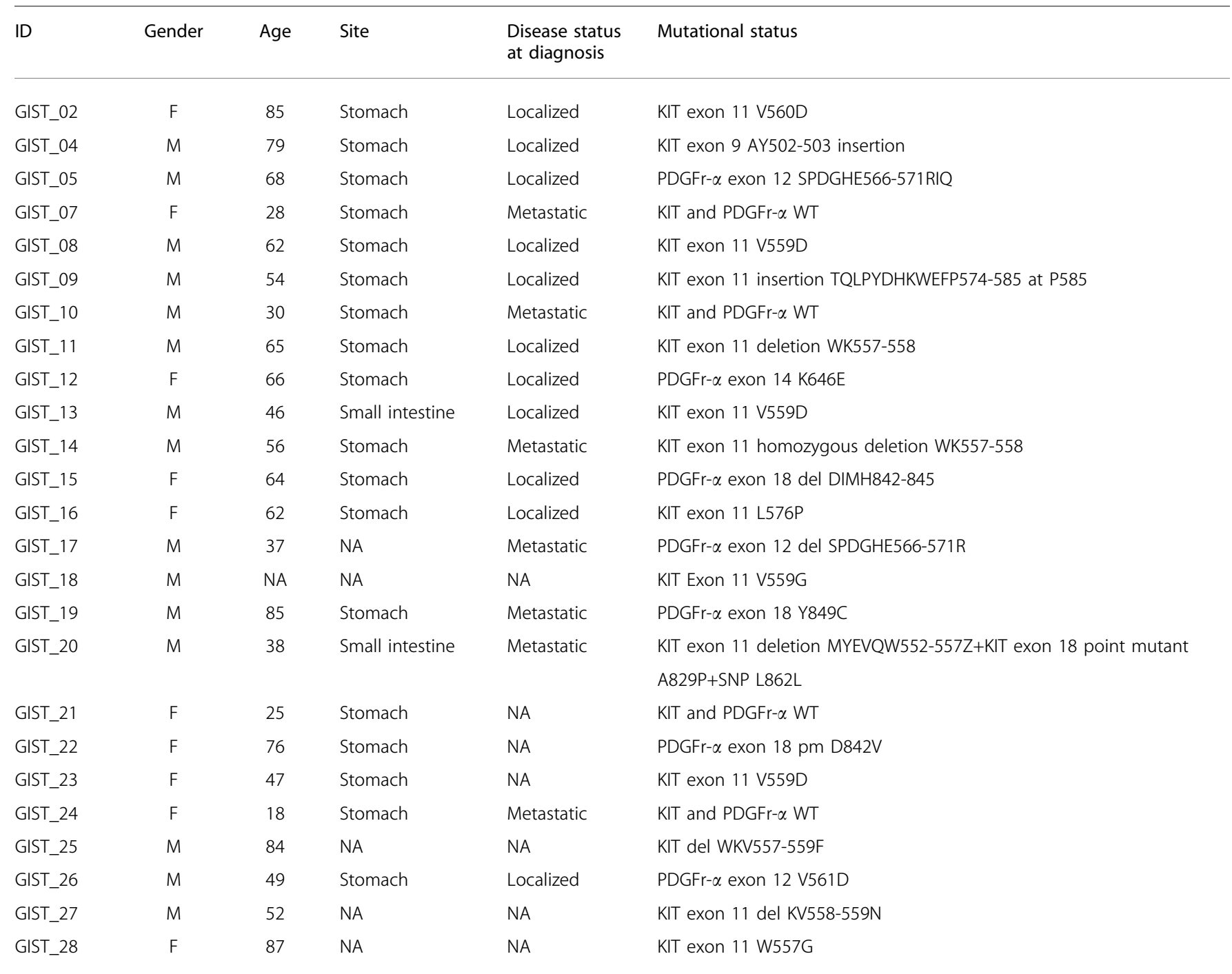

chromosome gains and losses. Twenty-one patients showed both macroscopic and cryptic cytogenetic alterations, whereas four patients (three of four WT) showed no genomic imbalances. The mean number of CNAs per patient was $10.76 \pm 2.73$, whereas the mean number of chromosomes harboring at least one alteration was $6.44 \pm 1.3$. Deletions outnumbered amplifications nearly threefold. The cytogenetic profiles were highly heterogeneous (Figure 1b). In particular, although the majority of patients showed a mean of four CNAs, six patients showed a very complex karyotype, averaging 17 chromosomes altered and an abnormally high number of CNAs (32.1 mean CNAs; $>75 \%$ percentile of the distribution; Supplementary Figure S1). All the chromosomes showed at least one CNA, with the most frequently altered chromosomes including chromosome 14 (17 of 25 patients), chromosome 1 (14 of 25 patients) and chromosome 22 (10 of 25 patients) (Figure 1a). Although WT patients did not show any alteration of chromosome 14, it was consistently altered in nearly all patients bearing a KIT or PDGFRA mutation (17 of 21 patients), either as an entire arm loss (monosomy) or as an interstitial deletion. Deletions on chromosome 14 showed various overlapping regions spanning inside cytobands 14q21.3, q23.1, q23.3-q31.3, q32.12 and q32.2-q32.33 (Supplementary Figure S2).

\section{Identification of Potential Oncogenes and Tumor Suppressors in GIST}

To pinpoint the genetic alterations beyond KIT and PDGFRA that promote GIST pathogenesis, we integrated whole-genome gene expression with copy number data on 22 of 25 patients. This approach identifies gene expression promoted directly by copy number state, thus narrowing the list of potential oncogenic events by excluding those genes whose expression is not influenced by the cytogenetic alteration. 
a
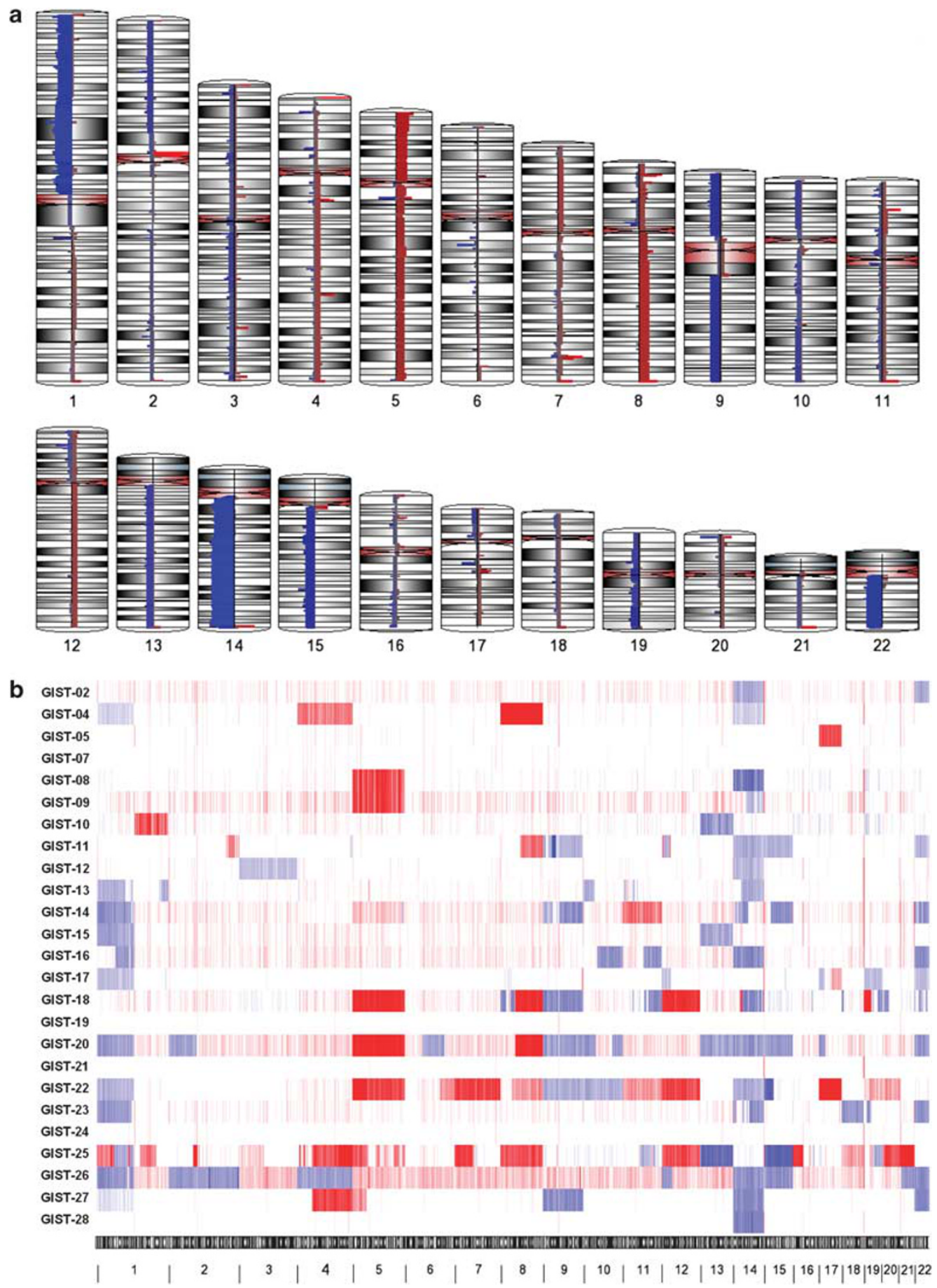

Figure 1 (a) Genomic copy number alterations in GIST samples. The frequency of each CNA is shown as a histogram (blue, deletion; red, amplification) inside each chromosome. The height of the histogram is proportional to the number of samples showing the CNA in each chromosomal region.

(b) Heatmap representation of copy number state: genomic deletions (blue) and amplifications (red) in 25 GIST samples.

A high level of correlation between CNAs and the correspondent gene expression profile was observed, with the mean correlation coefficient of 0.115 , significantly higher than the value expected by chance $(P<0.0001$; Supplementary Figure S3). To search for common oncogenic events in multiple samples, we first selected genomic regions amplified or deleted in more than four samples. These regions were cross-referenced with genes that map to the area and to the CancerGene database. Only those genes that showed a high correlation between gene expression and copy number state 
were selected ( $P$-value of correlation $<0.05)$. As expected, amplified oncogenes were less frequently observed as compared with deleted tumor suppressor genes (Tables 2 and 3 ). KRAS was identified as the promoter oncogene related to 12p12.1 amplification (4 of 22 patients), whereas many genes (GLI3, RALA and IGFBP3) are functionally related to $7 \mathrm{p}$ amplification. No significant correlation was observed between c-MYC expression and 8q amplification, even if the number of samples analyzed suggests to interpret negative findings with caution. Gene expression and copy number data integration allowed the identification of many haploinsufficient tumor suppressor genes linked to a $1 p$ deletion found in almost half of the samples. In particular, inside region $1 \mathrm{p} 36$, many tumor suppressor genes (KIF1B, UBE4B, DNAJC11, PRDM2 and TP73) were significantly underexpressed. Chromosome $9 \mathrm{p}$ deletion showed a peak region on $9 \mathrm{p} 21.3$, where CDKN2A and 2B map, but the strongest correlation with gene expression was found with the nearby MTAP gene. CDKN2A and $2 \mathrm{~B}$ showed significant expression silencing when associated with a focal event of homozygous deletion (three patients; data not shown), suggesting an involvement in GIST tumorigenesis that would deserve a deeper analysis in a wider sample data set. Chromosome 14 showed a common deletion region on the $14 \mathrm{q} 23.1$ cytoband, linked to a marked downregulation of PPM1A tumor suppressor gene, whereas loss of chromosome $22 \mathrm{q}$ was associated with NF2 underexpression.

\section{Identification of Shared Alterations in Mutated GIST}

To identify new oncogenic alterations promoting pathogenesis in KIT- or PDGFRA-mutated GIST samples, we first identified the regions of chromosomal aberration more frequently associated with the mutated phenotype. Significant regions $(P<0.05)$ were found only on chromosomes 1 and 14. The most significant association was in the q23.1-qter region of chromosome 14, with peak significance located in a region on 14q23.1 cytoband (Figure 2). To identify candidate genes in these regions, we merged copy number data with gene expression data, selecting only genes differentially expressed in WT vs mutated samples $(P<0.05)$. The majority of these genes ( 71 of $74,96 \%$ ) were downregulated in mutated samples (Figure 3). Not surprisingly, many of these genes have a role in cancer progression, such as DAAM1, RTN1, PPM1A, DACT1, MPP5, SNW1, FOXN3 and PTPN21 (Table 4). RTN1, DAAM1 and DACT1, located in the q23.1 cytoband, showed the strongest downregulation in mutant samples. Differential expression of select candidate genes was validated by qPCR. Notably, the genes located in the q23.1 region, particularly $R T N 1, D A A M 1$ and $D A C T 1$, were confirmed as showing the highest separation with more than fourfold expression difference (Figure 4). These genes are of high interest as they have a key role in the control of apoptosis and the WNT7/ $\beta$-catenin pathway. In particular RTN1 seems an interesting candidate target gene in GIST because of its well-documented role in the induction of apoptosis. ${ }^{23,24}$

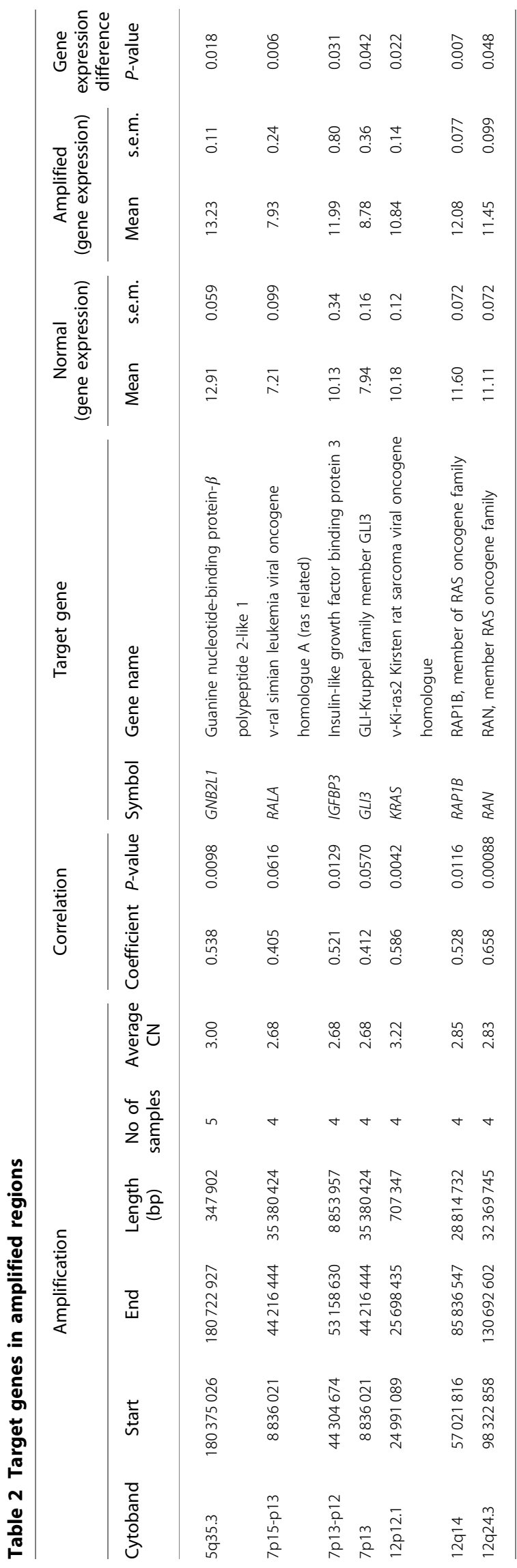


Table 3 Target genes in deleted regions

\begin{tabular}{|c|c|c|c|c|c|c|c|c|c|c|c|c|c|c|}
\hline \multicolumn{6}{|c|}{ Deletion } & \multicolumn{2}{|c|}{ Correlation } & \multicolumn{2}{|r|}{ Target gene } & \multicolumn{2}{|c|}{$\begin{array}{c}\text { Normal } \\
\text { (gene expression) }\end{array}$} & \multicolumn{2}{|c|}{$\begin{array}{c}\text { Deleted } \\
\text { (gene expression) }\end{array}$} & \multirow{2}{*}{$\begin{array}{c}\text { Gene ex- } \\
\text { pression } \\
\text { difference } \\
P \text {-value }\end{array}$} \\
\hline Cytoband & Start & End & $\begin{array}{l}\text { Length } \\
\text { (bp) }\end{array}$ & $\begin{array}{c}\text { no } \\
\text { samples }\end{array}$ & $\begin{array}{l}\text { Average } \\
\text { CN }\end{array}$ & Coefficient & $P$-value & Symbol & Gene name & Mean & s.e.m. & Mean & s.e.m. & \\
\hline $1 \mathrm{p} 36.31$ & 6198429 & 9266679 & 3068251 & 10 & 1.33 & 0.816 & $3.68 \mathrm{E}-06$ & DNAJC11 & DnaJ (Hsp40) homologue, subfamily C, member 11 & 7.44 & 0.059 & 6.91 & 0.048 & $<0.0001$ \\
\hline $1 \mathrm{p} 36.3$ & 9266679 & 10125743 & 859065 & 11 & 1.33 & 0.806 & $6.021 \mathrm{E}-06$ & UBE4B & Ubiquitination factor E4B (UFD2 homologue, yeast) & 8.95 & 0.10 & 7.85 & 0.13 & $<0.0001$ \\
\hline $1 p 36.2$ & 10203297 & 10309880 & 106584 & 11 & 1.33 & 0.780 & $1.88 \mathrm{E}-05$ & KIF1B & Kinesin family member $1 \mathrm{~B}$ & 9.12 & 0.084 & 8.22 & 0.16 & $<0.0001$ \\
\hline $1 \mathrm{p} 34$ & 32042489 & 33166505 & 1124017 & 11 & 1.31 & 0.749 & $5.988 \mathrm{E}-05$ & HDAC1 & Histone deacetylase 1 & 10.57 & 0.067 & 9.99 & 0.10 & $<0.0001$ \\
\hline $1 \mathrm{p} 36.21$ & 13416225 & 14273631 & 857407 & 10 & 1.31 & 0.706 & 0.00024 & PRDM2 & PR domain containing 2, with ZNF domain & 7.31 & 0.066 & 6.72 & 0.087 & $<0.0001$ \\
\hline $1 \mathrm{p} 36.3$ & 1982803 & 5943397 & 3960595 & 10 & 1.33 & 0.544 & 0.0089 & TP73 & Tumor protein p73 & 5.95 & 0.043 & 5.77 & 0.042 & 0.012 \\
\hline 1p13.2 & 112507584 & 113560589 & 1053006 & 12 & 1.32 & 0.413 & 0.056 & ST7L & Suppression of tumorigenicity 7 like & 5.76 & 0.063 & 5.47 & 0.073 & 0.007 \\
\hline $9 p 22.3$ & 1571783 & 5265163 & 3693381 & 7 & 1.27 & 0.727 & 0.00013 & SMARCA2 & $\begin{array}{l}\text { SWI/SNF-related, matrix-associated, actin-dependent } \\
\text { regulator of chromatin, subfamily a, member } 2\end{array}$ & 8.10 & 0.099 & 7.16 & 0.14 & $<0.0001$ \\
\hline $9 \mathrm{p} 21$ & 21765976 & 21802029 & 36054 & 8 & 1.19 & 0.619 & 0.0021 & MTAP & Methylthioadenosine phosphorylase & 5.17 & 0.095 & 4.49 & 0.22 & 0.002 \\
\hline $9 \mathrm{p} 21$ & 30260414 & 33783156 & 3522743 & 7 & 1.33 & 0.598 & 0.0033 & TOPORS & Topoisomerase I binding, arginine/serine-rich & 8.33 & 0.078 & 7.60 & 0.24 & 0.001 \\
\hline $9 \mathrm{q} 31$ & 84595472 & 106721870 & 22126399 & 7 & 1.27 & 0.548 & 0.0082 & TMEFF1 & $\begin{array}{l}\text { Transmembrane protein with EGF-like and two } \\
\text { follistatin-like domains } 1\end{array}$ & 5.01 & 0.24 & 3.87 & 0.10 & 0.009 \\
\hline $13 q 12.2$ & 22536202 & 31430096 & 8893895 & 4 & 1.12 & 0.815 & $3.893 \mathrm{E}-06$ & RNF6 & Ring-finger protein ( $\mathrm{C}^{2} \mathrm{H} 2 \mathrm{C} 3$ type) 6 & 9.50 & 0.067 & 8.60 & 0.060 & $<0.0001$ \\
\hline $13 q 11-q 12$ & 19198237 & 22523922 & 3325686 & 4 & 1.12 & 0.671 & 0.00062 & LATS2 & $\begin{array}{l}\text { LATS, large tumor suppressor, homologue } 2 \\
\text { (Drosophila) }\end{array}$ & 10.33 & 0.15 & 9.31 & 0.11 & 0.006 \\
\hline $\begin{array}{l}13 q 14.12- \\
q 14.2\end{array}$ & 48011276 & 54590729 & 6579454 & 4 & 1.15 & 0.641 & 0.0013 & INTS6 & Integrator complex subunit 6 & 8.85 & 0.14 & 8.15 & 0.18 & 0.036 \\
\hline $13 q 14$ & 48011276 & 54590729 & 6579454 & 4 & 1.15 & 0.632 & 0.0016 & TRIM13 & Tripartite motif-containing 13 & 7.41 & 0.14 & 6.10 & 0.37 & 0.0006 \\
\hline $14 \mathrm{q} 21.1$ & 38585823 & 38760942 & 175120 & 14 & 1.26 & 0.791 & $1.168 \mathrm{E}-05$ & $P N N$ & Pinin, desmosome-associated protein & 9.73 & 0.15 & 8.79 & 0.13 & 0.0002 \\
\hline $14 \mathrm{q} 23.1$ & 59284472 & 61286479 & 2002008 & 16 & 1.24 & 0.710 & 0.00022 & PPM1A & Protein phosphatase $1 A \alpha$ isoform & 8.52 & 0.12 & 7.15 & 0.13 & $<0.0001$ \\
\hline $14 q 24.3-q 31$ & 73126255 & 89981048 & 16854794 & 16 & 1.24 & 0.552 & 0.0078 & SEL1L & sel-1 suppressor of lin-12-like (C. elegans) & 9.48 & 0.097 & 9.00 & 0.089 & 0.005 \\
\hline $15 q 15-q 21$ & 38623562 & 47871023 & 9247462 & 7 & 1.17 & 0.785 & $1.487 \mathrm{E}-05$ & TP53BP1 & Tumor protein p53 binding protein, 1 & 9.32 & 0.080 & 8.55 & 0.10 & $<0.0001$ \\
\hline $15 q 22.1$ & 59487911 & 62137778 & 2649868 & 7 & 1.29 & 0.505 & 0.0164 & TPM1 & Tropomyosin $1(\alpha)$ & 11.24 & 0.20 & 9.72 & 0.21 & 0.0001 \\
\hline $15 q 22$ & 63638766 & 74646820 & 11008055 & 7 & 1.29 & 0.487 & 0.0214 & PML & Promyelocytic leukemia & 6.10 & 0.097 & 5.21 & 0.33 & 0.003 \\
\hline $22 q 12.2$ & 26945773 & 30145210 & 3199438 & 10 & 1.21 & 0.794 & 1.007E-05 & NF2 & Neurofibromin 2 (bilateral acoustic neuroma) & 8.25 & 0.076 & 7.60 & 0.061 & $<0.0001$ \\
\hline $22 q 11.23$ & 21593265 & 22607380 & 1014116 & 9 & 1.25 & 0.594 & 0.0035 & SMARCB1 & $\begin{array}{l}\text { SWI/SNF-related, matrix-associated, actin-dependent } \\
\text { regulator of chromatin, subfamily b, member } 1\end{array}$ & 8.56 & 0.045 & 7.98 & 0.11 & $<0.0001$ \\
\hline
\end{tabular}




\section{a}

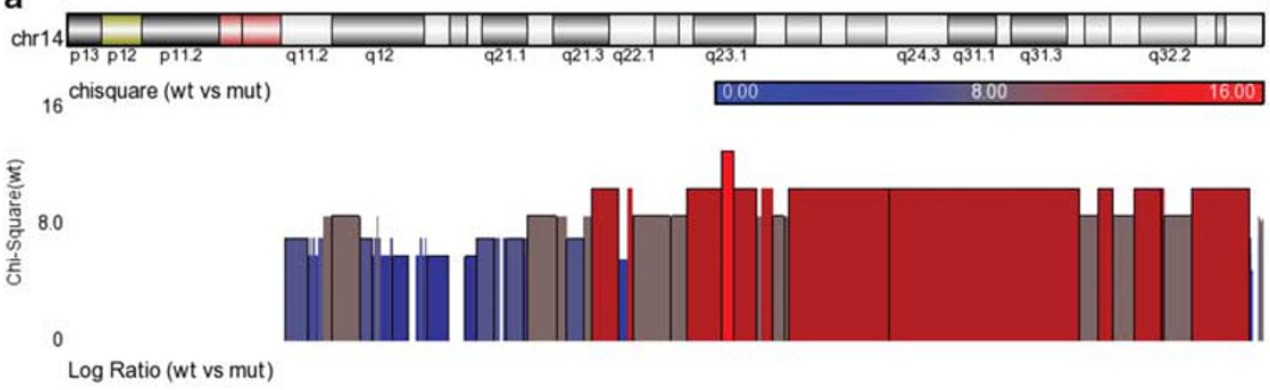

b

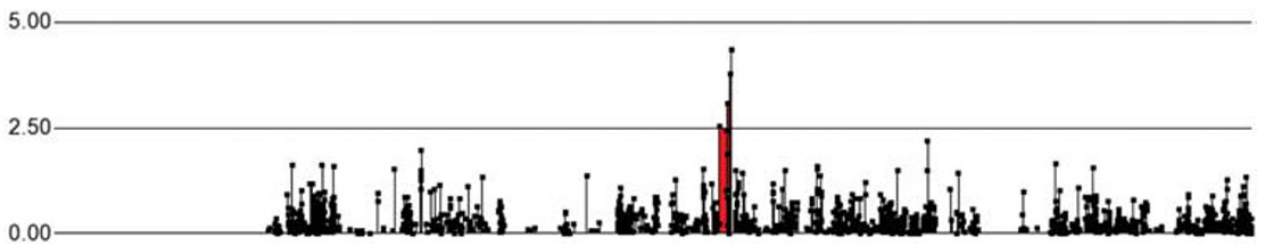

Figure 2 Integration of CNA and differential gene expression on chromosome 14. (a) $\chi^{2}$-Analysis of the association of a specific copy number alteration to the WT or mutated samples class. Higher histogram height and red vs blue represent greater association; (b) gene expression log ${ }_{2}$ ratio in WT samples regarding mutant samples. The red area under the curve represents the highest gene expression fold change.

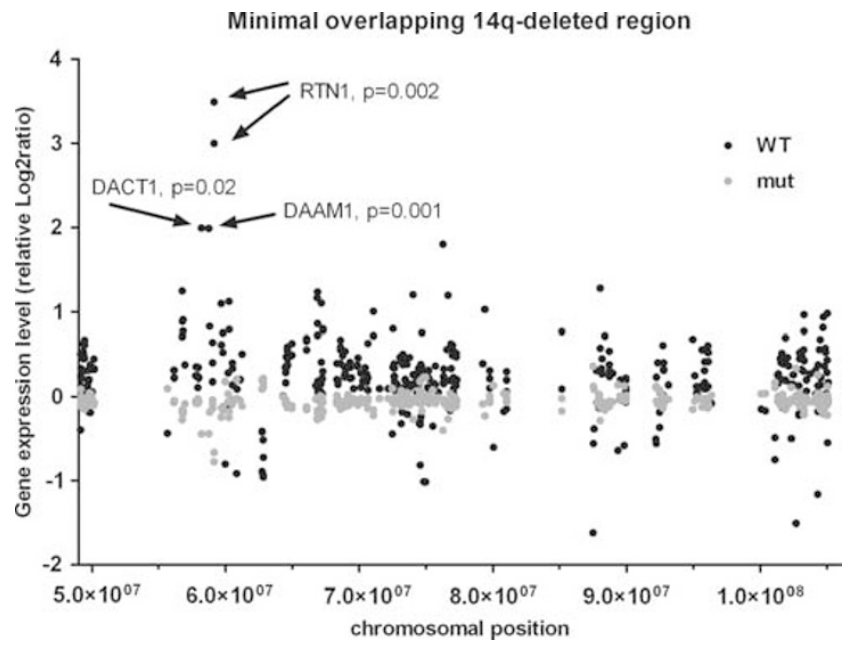

Figure 3 Scatter plot representation of average gene expression in WT (black) and mutant (gray) samples in the minimal overlapping region on chr14 deleted in 16 of 22 samples. The maximal gene expression difference and statistical significance is found in the region on $14 q 23.1$ cytoband that harbors RTN1, DAAM1 and DACT1 genes.

Indeed complete inhibition of its expression in GIST882 cell line by siRNA determined a significant increase in cell growth regarding nontargeting siRNA-transfected cells (Figure 5).

\section{DISCUSSION}

Until recently, only abnormalities of KIT and PDGFRA were identified as molecular events underlying GIST development, and as the only target of medical therapies. However, sequential accumulation of other genetic events besides KIT and PDGFRA mutations may be involved in GIST development and progression. The significance of these changes has not been thoroughly investigated. ${ }^{7}$ A comprehensive and integrative analysis of gene expression profiling and highresolution genomic copy number could improve our understanding of GIST tumor development and progression. Here, we report the first study integrating gene expression profiling and high-resolution genomic copy number analyses in GISTs.

Of 25, 21 patients showed both macroscopic cytogenetic alterations and cryptic microdeletions or amplifications by SNP array copy number data analysis, whereas the remaining 4 patients showed no genomic imbalances. Cytogenetic profiling of each sample was extremely heterogeneous in that a high variability of alterations both per chromosome and per patient was observed. The most frequently observed chromosomal alterations were $14 \mathrm{q}$ complete or partial deletion (17 of 25), $1 \mathrm{p}$ deletion (14 of 25) and 22q deletion (10 of 25).

Chromosome 14 was consistently altered in almost all patients bearing KIT or PDGFRA mutations (17 of 21), either as a whole chromosome arm loss (monosomy) or as an interstitial deletion. WT patients did not show any alteration in chromosome 14. Previous studies determined losses of $14 \mathrm{q}$ and $22 \mathrm{q}$ are the most common cytogenetic abnormalities in GISTs, followed by loss of 1p, 9p, 15q, 13q. This suggests that these genetics aberrations may be important in both early tumor development and progression. ${ }^{8-20}$ Gunawan et $a l^{8}$ have recently proposed an oncogenetic tree model identifying three major cytogenetic pathways in 203 primary GISTs: one initiated by $-14 \mathrm{q}$, one by $-1 \mathrm{p}$ and another by $-22 \mathrm{q}$, with different biologic and clinical behavior. GISTs involving the $-14 \mathrm{q}$ pathway were predominantly gastric tumors with stable karyotype and more favorable clinical course. GISTs involving the $-1 \mathrm{p}$ pathway were mostly intestinal GISTs, with a greater cytogenetic complexity and more aggressive 
clinical course. Finally, GISTs involving the $-22 \mathrm{q}$ were both gastric and intestinal tumors, with a complex karyotype and unfavorable clinical outcome. ${ }^{8}$ In our study, all mutant patients with a complex karyotype showed loss of 14q, usually accompanied by $1 \mathrm{p}$ and $22 \mathrm{q}$ deletions. Notably, many of the patients showing a nearly stable karyotype showed a $14 \mathrm{q}$ deletion.

Assämäki et al ${ }^{9}$ identified two recurrent deleted regions at $14 \mathrm{q}$ harboring genes involved in DNA repair, tumor suppression and apoptosis regulation, such as PARP2, APEX1, NDRG2 and SIVA. Furthermore, they suggest other target candidates, such as NF2 at chromosome $22, \mathrm{CDKN} 2 \mathrm{~A} / 2 \mathrm{~B}$ at $9 \mathrm{p}$ and ENO1 at $1 \mathrm{p} .{ }^{9}$ Here, we support these findings by correlating gene expression and CNAs, confirming that 22q deletion leads to NF2 downregulation, and that $9 \mathrm{p}$ deletion is linked to CDKN2A and $2 \mathrm{~B}$ inactivation only when a focal event of homozygous deletion is present. CDKN2A and 2B encode inhibitors of cell-cycle kinases, and their role in GIST progression has been previously investigated. ${ }^{25-27}$

All GIST patients with mutant KIT or PDGFRA showed a wide range of cytogenetic alterations, whereas three of four WT GIST patients showed no genomic imbalances. Wozniak et $a l^{10}$ reported that the genomic profile of GISTs bearing PDGFRA and KIT mutations seems to be independent from the tumor mutational status. Consistent with our results, Belinsky et $a l^{28}$ reported that most WT GISTs show few or no genomic alterations. Taken together, this suggests that adult WT GISTs show a minimal cytogenetic progression in comparison with mutant GISTs. The lack of genomic imbalances in WT GISTs suggests a mechanism beside these cytogenetic alterations have in tumorigenesis. We recently reported that WT GISTs overexpress IGF-1R, in spite of the SNP array gene copy analysis determined none of the patients bore IGF-1R amplification. ${ }^{29}$ Similarly, previous studies have suggested that both adult and pediatric WT GISTs may share alterations in IGF-1 pathway. ${ }^{30-33}$

By merging copy number and gene expression data, we observed a high level of correlation between CNAs and the correspondent gene expression profile. This was especially true on chromosome 14, in the q23.1-qter region, which harbors several possible target genes (DAAM1, RTN1, PPM1A, DACT1, MPP5, SNW1, FOXN3, PPM1A and PTPN21). Differential expression between mutant and WT samples was validated by qPCR, with the highest separation observed in the repressed expression of RTN1, DAAM1 and $D A C T 1$ in the mutant samples. These genes are strong candidates as tumor suppressors in the development and progression of GISTs harboring mutations in KIT or PDGFRA. $D A C T 1$ negatively modulates the basal activity of $\mathrm{WNT} / \beta$ catenin signaling both in the cytoplasm and the nucleus, suggesting an important role in embryogenesis and cancer development by regulating the expression of genes involved in cell proliferation, differentiation and survival. ${ }^{34,35}$ RTN1 belongs to the family of reticulum encoding genes and is involved in different apoptosis pathways, ${ }^{23,24}$ and we showed 

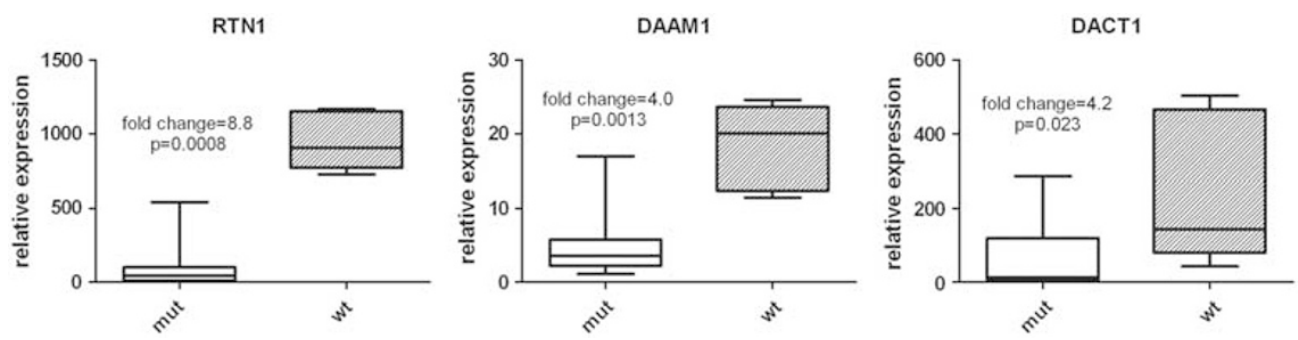

Figure 4 Boxplot representation of gene expression levels measured by qPCR of candidate target genes in WT and mutated samples.

a

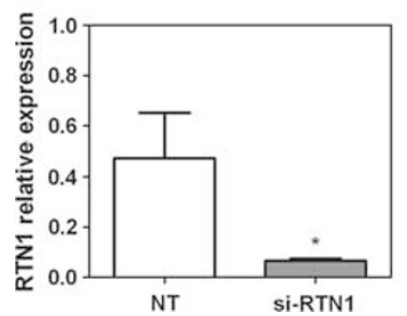

b

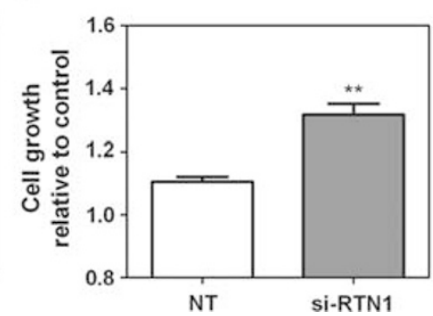

Figure 5 siRNA-mediated RTN1 knockdown in GIST882 cells. (a) Inhibition of RTN1 expression by siRNA regarding nontargeting siRNA (NT) by means of qPCR. (b) Effect of RTN1 silencing on GIST882 cell growth after $72 \mathrm{~h}$ of incubation, regarding nontargeting siRNA (NT) $\left({ }^{*} P<0.05, * * P<0.01\right.$, Mann-Whitney U-test).

here that its inhibition significantly increases cell growth of GIST cell line 882 over nontargeting control siRNA, providing further evidence on the effective identification of target haploinsufficient genes involved in GIST pathogenesis. In conclusion, we report the first study integrating genomewide copy number and gene expression data obtained by microarray analyses in GIST. The cytogenetic profile of GISTs is highly heterogeneous and is affected by mutational status. In particular, a marked difference was noted in the karyotypes of mutant and WT GIST samples. Furthermore, a high level of correlation between CNAs and the correspondent gene expression profile was observed. The combination of these two techniques has allowed a complete and detailed molecular portrait of GIST to emerge. This portrait highlighted the putative role of known and novel oncogenes and tumor suppressor genes whose further analysis is required for a comprehensive knowledge of tumor pathogenesis and to discover novel molecular targets in GISTs.

Supplementary Information accompanies the paper on the Laboratory Investigation website (http://www.laboratoryinvestigation.org)

\section{ACKNOWLEDGEMENTS}

Research programs on GISTs are supported by Fondazione Cassa di Risparmio of Bologna (CARISBO), Fondazione Giuseppe Alazio, Palermo, and Vanini-Cavagnino grant. This research was also supported in part by A VA Merit Review grant $(\mathrm{MCH})$ and funding from the Life Raft Group (MCH).

\section{DISCLOSURE/CONFLICT OF INTEREST}

The authors declare no conflict of interest.

1. Hirota $S$, Isozaki K, Moriyama $Y$, et al. Gain of function mutations of c-kit in human gastrointestinal stromal tumors. Science 1998;279: 577-580.

2. Heinrich $\mathrm{MC}$, Corless $\mathrm{CL}$, Duensing $\mathrm{A}$, et al. PDGFRA activating mutations in gastrointestinal stromal tumors. Science 2003;299: 708-710.

3. Medeiros F, Corless CL, Duensing A, et al. KIT-negative gastrointestinal stromal tumors. Am J Surg Pathol 2004;28:889-894.

4. Heinrich MC, Corless CL, Demetri GD, et al. Kinase mutations and imatinib response in patients with metastatic gastrointestinal stromal tumor. J Clin Oncol 2003;21:4342-4349.

5. Debiec-Rychter M, Dumez H, Judson I, et al. Use of c-KIT/PDGFRA mutational analysis to predict the clinical response to imatinib in patients with advanced gastrointestinal stromal tumors entered on phase I and II studies of the EORTC Soft Tissue and Bone Sarcoma Group. Eur J Cancer 2004;40:689-695.

6. Maleddu A, Pantaleo MA, Nannini M, et al. Mechanisms of secondary resistance to tyrosine kinase inhibitors in gastrointestinal stromal tumors (Review). Oncol Rep 2009;21:1359-1366.

7. Yang J, Du X, Lazar AJ, et al. Genetic aberrations of gastrointestinal stromal tumors. Cancer 2008;113:1532-1543.

8. Gunawan B, von Heydebreck A, Sander B, et al. An oncogenetic tree model in gastrointestinal stromal tumours (GISTs) identifies different pathways of cytogenetic evolution with prognostic implications. J Pathol 2007;211:463-470.

9. Assämäki R, Sarlomo-Rikala $M$, Lopez-Guerrero JA, et al. Array comparative genomic hybridization analysis of chromosomal imbalances and their target genes in gastrointestinal stromal tumors. Genes Chromosomes Cancer 2007;46:564-576.

10. Wozniak A, Sciot R, Guillou $L$, et al. Array CGH analysis in primary gastrointestinal stromal tumors: cytogenetic profile correlates with anatomic site and tumor aggressiveness, irrespective of mutational status. Genes Chromosomes Cancer 2007;46:261-276.

11. Chen $Y$, Tzeng CC, Liou CP, et al. Biological significance of chromosomal imbalance aberrations in gastrointestinal stromal tumors. J Biomed Sci 2004;11:65-71.

12. Breiner JA, Meis-Kindblom J, Kindblom LG, et al. Loss of 14q and 22q in gastrointestinal stromal tumors (pacemaker cell tumors). Cancer Genet Cytogenet 2000;120:111-116.

13. Debiec-Rychter $M$, Sciot $R$, Pauwels $P$, et al. Molecular cytogenetic definition of three distinct chromosome arm 14q deletion intervals in gastrointestinal stromal tumors. Genes Chromosomes Cancer 2001; 32:26-32.

14. Derre J, Lagach R, Terier $\mathrm{P}$, et al. Consistent loss on the short arm of chromosome 1 in a series of malignant gastrointestinal stromal tumors. Cancer Genet Cytogenet 2001;127:30-33.

15. El-Rifai W, Sarlomo-Rikala M, Andersson LC, et al. DNA sequence copy number changes in gastrointestinal stromal tumors: tumor progression and prognostic significance. Cancer Res 2000;60:3899-3903.

16. El-Rifai W, Sarlomo-Rikala M, Andersson LC, et al. High resolution deletion mapping of chromosome 14 in stromal tumors of the gastrointestinal tract suggests two distinct tumor suppressor loci. Genes Chromosomes Cancer 2000;27:387-391. 
17. El-Rifai W, Sarlomo-Rikala M, Miettinen $M$, et al. DNA copy number losses in chromosome 14: an early change in gastrointestinal stromal tumors. Cancer Res 1996:56:3230-3233.

18. Chen $\mathrm{Y}$, Liou CP, Tseng HH, et al. Deletions of chromosome $1 \mathrm{p}$ and $15 \mathrm{q}$ are associated with aggressiveness of gastrointestinal stromal tumors. J Formos Med Assoc 2009;108:28-37.

19. Lasota J, Wozniak A, Kopczynski J, et al. Loss of heterozygosity on chromosome $22 q$ in gastrointestinal stromal tumors (GISTs): a study on 50 cases. Lab Invest 2005;85:237-247.

20. Kim NG, Kim JJ, Ahn JY, et al. Putative chromosomal deletions on $9 p$ $9 q$ and $22 q$ occur preferentially in malignant gastrointestinal stromal tumors. Int J Cancer 2000;85:633-638.

21. Tibshirani R, Wang P. Spatial smoothing and hot spot detection for CGH data using the fused lasso. Biostatistics 2008;9:18-29.

22. Smyth GK. Linear models and empirical Bayes methods for assessing differential expression in microarray experiments. Stat Appl Genet Mo Biol 2004;3 Article 3.

23. Di Sano F, Fazi B, Tufi R, et al. Reticulon-1C acts as a molecular switch between endoplasmic reticulum stress and genotoxic cell death pathway in human neuroblastoma cells. J Neurochem 2007; 102:345-353.

24. Fazi B, Melino S, De Rubeis S, et al. Acetylation of RTN-1C regulates the induction of ER stress by the inhibition of HDAC activity in neuroectodermal tumors. Oncogene 2009;28:3814-3824.

25. Schneider-Stock R, Boltze C, Lasota J, et al. Loss of p16 protein defines high-risk patients with gastrointestinal stromal tumors: a tissue microarray study. Clin Cancer Res 2005;11:638-645.

26. Sabah $M$, Cummins $R$, Leader $M$, et al. Altered expression of cell cycle regulatory proteins in gastrointestinal stromal tumors: markers with potential prognostic implications. Hum Pathol 2006;37:648-655.

27. Schmieder M, Wolf S, Danner B, et al. p16 expression differentiates high-risk gastrointestinal stromal tumor and predicts poor outcome. Neoplasia 2008;10:1154-1162.

28. Belinsky MG, Skorobogatko $Y$, Rink $L$, et al. High density DNA array analysis reveals distinct genomic profiles in a subset of gastrointestinal stromal tumors. Genes Chromosomes Cancer 2009;48:886-896.

29. Pantaleo MA, Astolfi A, Di Battista M, et al. Insulin-like growth factor 1 receptor expression in wild-type GISTs: a potential novel therapeutic target. Int J Cancer 2009;125:2991-2994.

30. Prakash S, Sarran L, Socci N, et al. Gastrointestinal stromal tumors in children and young adults: a clinicopathologic, molecular, and genomic study of 15 cases and review of the literature. J Pediatr Hematol Oncol 2005;27:179-187.

31. Braconi C, Bracci R, Bearzi I, et al. Insulin-like growth factor (IGF) 1 and 2 help to predict disease outcome in GIST patients. Ann Oncol 2008;19:1293-1298.

32. Belinsky MG, Rink $L$, Cai KQ, et al. The insulin-like growth factor system as a potential therapeutic target in gastrointestinal stromal tumors. Cell Cycle 2008;7:2949-2955.

33. Tarn C, Rink L, Merkel E, et al. Insulin-like growth factor 1 receptor is a potential therapeutic target for gastrointestinal stromal tumors. Proc Natl Acad Sci USA 2008;105:8387-8392.

34. Gao X, Wen J, Zhang L, et al. Dapper1 is a nucleocytoplasmic shuttling protein that negatively modulates Wnt signaling in the nucleus. J Biol Chem 2008;283:35679-35688.

35. llyas M. Wnt signalling and the mechanistic basis of tumour development. J Pathol 2005;205:130-144. 